\title{
Adaptive Pulse Compression of MIMO Radar Based on SIC Ying-Hua Tian ${ }^{1, a^{*}}$, Xiu-Kun Ren ${ }^{1, b}$ and Xiao-Ying $\mathrm{Li}^{1, \mathrm{c}}$ \\ ${ }^{1}$ Zhengzhou Institute of Information Science and Technology, Zhengzhou, China \\ atian.ying.hua@foxmail.com, ${ }^{\text {c } r x k d l k @ 126 . c o m, ~}{ }^{b} 274845551 @ q q . c o m$
}

Keywords: MIMO Radar; Adaptive Pulse Compression; Successive Interference Cancellation

\begin{abstract}
Multiple-Input-Multiple-Output (MIMO) radar is recently the focus of radar research, which transmits different waveforms to illuminate the target from different antennas simultaneously. However the cross correlation of the waveforms may lead to interference between receiving signals. In this paper, we consider the use of adaptive pulse compression based on successive interference cancellation (SIC) to mitigate the mutual interference in MIMO radar receiver. The proposed method cancels interference successively on the pulse compression stage of each observation channel by subtracting the estimation of prior channel. The simulation results demonstrate the effectiveness of the proposed method.
\end{abstract}

\section{Introduction}

Multiple-Input-Multiple-Output (MIMO) radar [1,2] transmits different waveforms to obtain advantages such as larger virtual array, better estimation resolution over traditional phased-array radar. Therefore, MIMO radar receiver has to extract each waveforms from the receive signal which contains a group of overlapped transmit waveforms. Match filters are applied to recover each individual waveform as well as the target parameter. However, the cross correlation of the waveforms may lead to interference between multiple receiving signals.

Specially designed waveform set with good cross-correlation is used to solve the problem $[3,4]$. However, it is difficult to find those waveforms and the length of those waveform is still limited, which influences the receiver performance. Another approach is adaptive receiver filter $[5,6]$. But it always demands an algorithm complexity of $O\left(K^{3}\right)$ where $K$ is the length of filter and involves matrix inversion operation which is usually unstable.

In modem communication systems, especially the code-division multiple-access (CDMA) system, there is similar multiple-access interference (MAI) problem when several users communication using the same spectrum simultaneously, which can be solved by successive interference cancellation (SIC). In this paper, we introduce adaptive pulse compression based on SIC to cancel the interference in MIMO radar receiver and then apply numerical simulation to verify the effectiveness of the proposed method.

\section{System Model}

Fig. 1 shows the structure of MIMO radar used in this paper. For simplicity, we assume the range of the target is much larger than the length of transmit array and receiver array. The target is illuminated by $\mathrm{M}$ orthogonal signals transmitted by the transmit antennas. The transmitted baseband signals are denoted by $S_{m} \in C^{1 \times K}$, when $m$ denotes the $m$ th transmitter and $\mathrm{K}$ is the length of the signal. Assume that there is a target with direction of departures (DODs) $\theta$ and direction of arrivals (DOAs) $\varphi$. The signal received by the nth receiver can be written as

$$
\vec{x}_{n}=\sum_{i=1}^{M} \alpha_{i n} \vec{S}_{i}+\vec{w}_{n}
$$




$$
\alpha_{\text {in }}=a_{r}(\theta) a_{t}^{T}(\varphi) e
$$

$e \in C$ is the RCS of the target. $a_{r}(\theta) \in \mathrm{C}^{1 \times N}$ denotes the steering vector of receiver array and $a_{t}(\varphi) \in \mathrm{C}^{1 \times M}$ is the transmit array steering vector. $\alpha_{i n} \in C$ denotes the gain of observation channel between ith transmit antenna and $n$th receiver antenna. $\vec{w}_{n} \in C^{1 \times K}$ denotes noise in the receiver. We consider a jammer free model in which $\vec{w}_{n}$ is an independent Gaussian noise and $\vec{w} \vec{w}^{H}=\sigma^{2}$.

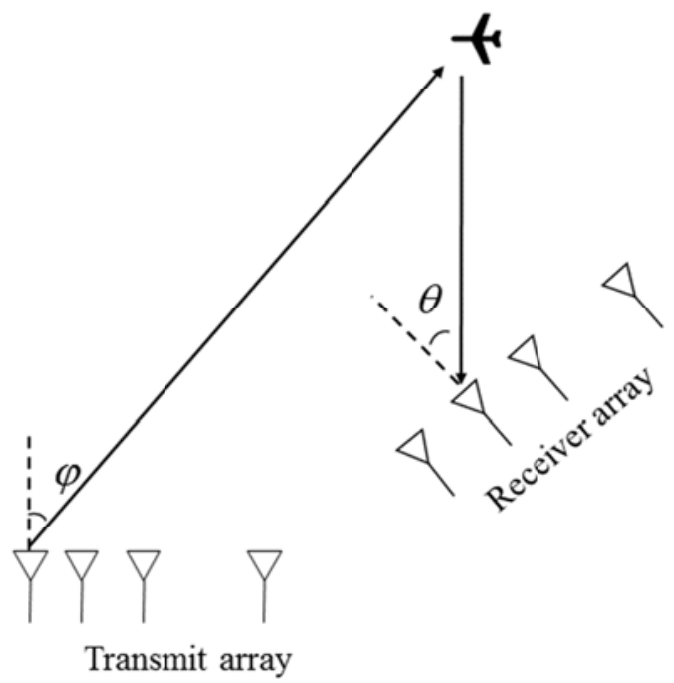

Fig.1. MIMO radar scenario.

\section{Adaptive Pulse Compression Based on SIC}

In this section, the SIC method was derived to improve the result of the pulse compression stage in MIMO radar receiver.

The result of conventional pulse compression can be described by the expression

$$
\vec{y}_{m n}=\vec{x}_{n} \vec{S}_{m}^{H}=\alpha_{m n} \vec{s}_{m} \vec{S}_{m}^{H}+\sum_{i \neq m} \alpha_{i n} \vec{s}_{i} \vec{s}_{m}^{H}+\vec{w}_{n} \vec{s}_{m}^{H}
$$

Where $\frac{1}{K} \sum_{i \neq m} \alpha_{i n} \vec{s}_{i} \vec{s}_{m}^{H} \quad$ is just the interference between transmit waveforms.

It is clear that we can cancel interference by removing the second term from right side of the Eq. 3 , which can be done by simply subtracting the estimation of this term from the equation. Furthermore, the interference could be eliminated even before the match filter as shown in Eq. 3, if the estimation of $\alpha_{i n}$ has been computed in the prior stage.

$$
\begin{aligned}
\vec{y}_{I C m n} & =\alpha_{m n} \vec{s}_{m} \vec{s}_{m}^{H}+\sum_{i \neq m} \alpha_{i n} \vec{s}_{i} \vec{s}_{m}^{H}+\vec{w}_{n} \vec{s}_{m}^{H}-\sum_{i \neq m} \hat{\alpha}_{i n} \vec{s}_{i} \vec{s}_{m}^{H} \\
& =\left(\vec{x}_{n}-\sum_{i \neq m} \hat{\alpha}_{i n} \vec{s}_{i}\right) \vec{s}_{m}^{H} \\
& =\vec{x}_{I C m n} \vec{s}_{m}^{H}
\end{aligned}
$$


Where

$$
\vec{x}_{I C m n}=\vec{x}_{n}-\sum_{i \neq m} \hat{\alpha}_{i n} \vec{s}_{i}
$$

In Eq. 4, $\vec{y}_{\text {ICmn }}$ denotes the output of pulse compression after the interference cancellation method and $\hat{\alpha}_{i n}$ denotes the initial estimate of $\alpha_{i n}$, which can be obtained on the prior stage.

$$
\hat{\alpha}_{i n}=\vec{x}_{n} \vec{s}_{i} / \vec{s}_{i} \vec{s}_{i}^{H}
$$

The whole signal flow of SIC method is illustrated in Fig. 2 in which the result of pulse compression on the prior stage is used to estimate the origin signal. The estimation are then subtracted from the input of succeeding pulse compression stages. This process of refining the output of match filter and then re-estimating the target profiles can be repeated for number of stages until all channels are extracted.

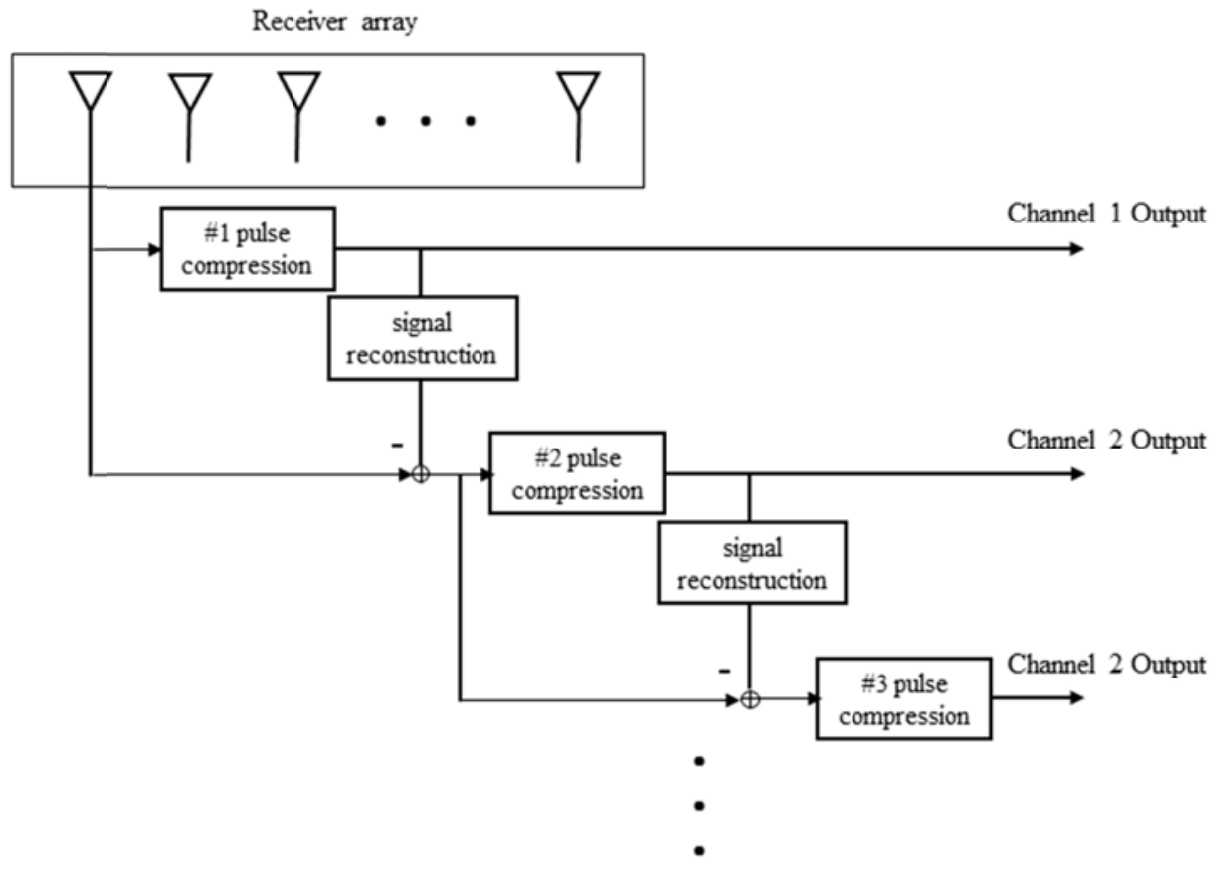

Fig.2. Signal Flow of Successive Interference Cancellation.

At each stage of the SIC process, the input signal is subtracted by the prior estimate of interference and then conventional pulse compression is applied to estimate channel gain. The total computation load, at the same level of conventional pulse compression algorithm, is proportional to $K^{2}$ when the adaptive filtering method demand a cost of $O\left(K^{3}\right)$ [5,7]. The method proposed is also of more stability for the lack of matrix inversion.

\section{Simulation Results}

It is assumed eight transmit antennas and eight receiver antenmas are placed with half-wavelength between each other. Each transmit antennas concurrently transmits a different waveform, Fig. 3 shows the auto-correlations and cross-correlation of transmitted waveforms, which is generated by polyphase coded Gold sequences with the length of 127 . 

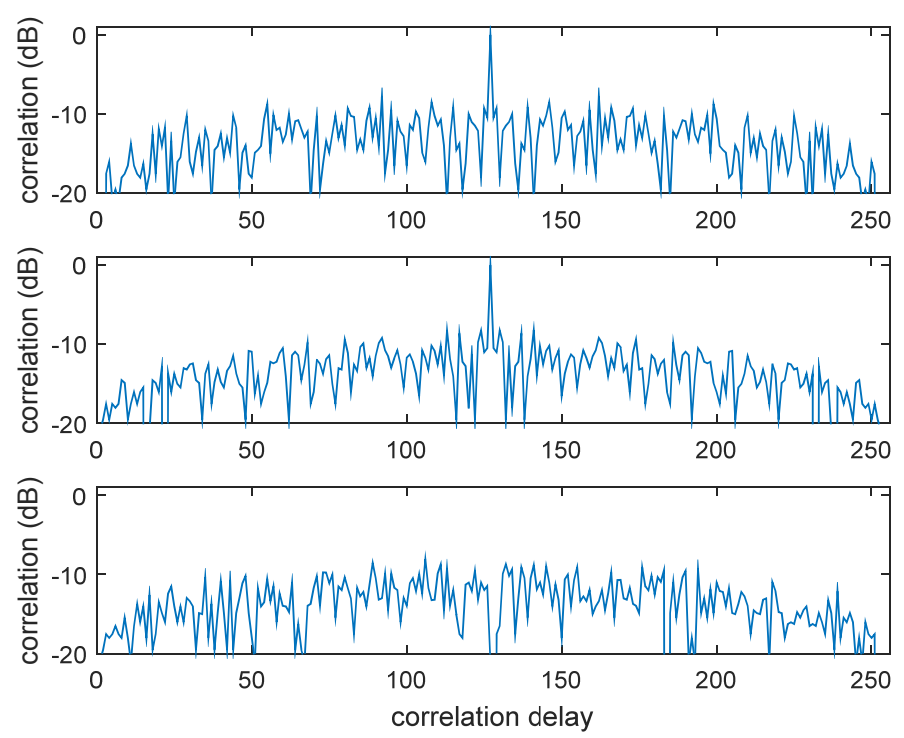

Fig.3. Correlations of transmitted waveforms.

Considering a still target is in the main beam of MIMO radar. The SNR level of received signal is $5 \mathrm{~dB}$. We compare the performance of adaptive pulse compression based on SIC (APC-SIC) with that of conventional pulse compression (PC) and the ground truth of the waveform's autocorrelation.

Fig. 4 illustrated the results for this scenario, in which the black line represents the ground truth of respective composite range profiles. As expected, the conventional pulse compression estimate is rather poor due to the interference of other transmitted signals, yielding a filter output with a $-5 \mathrm{~dB}$ peak sidelobe level. However the APC-SIC approach outperforms the match filtering with a $-15 \mathrm{~dB}$ peak sidelobe level.

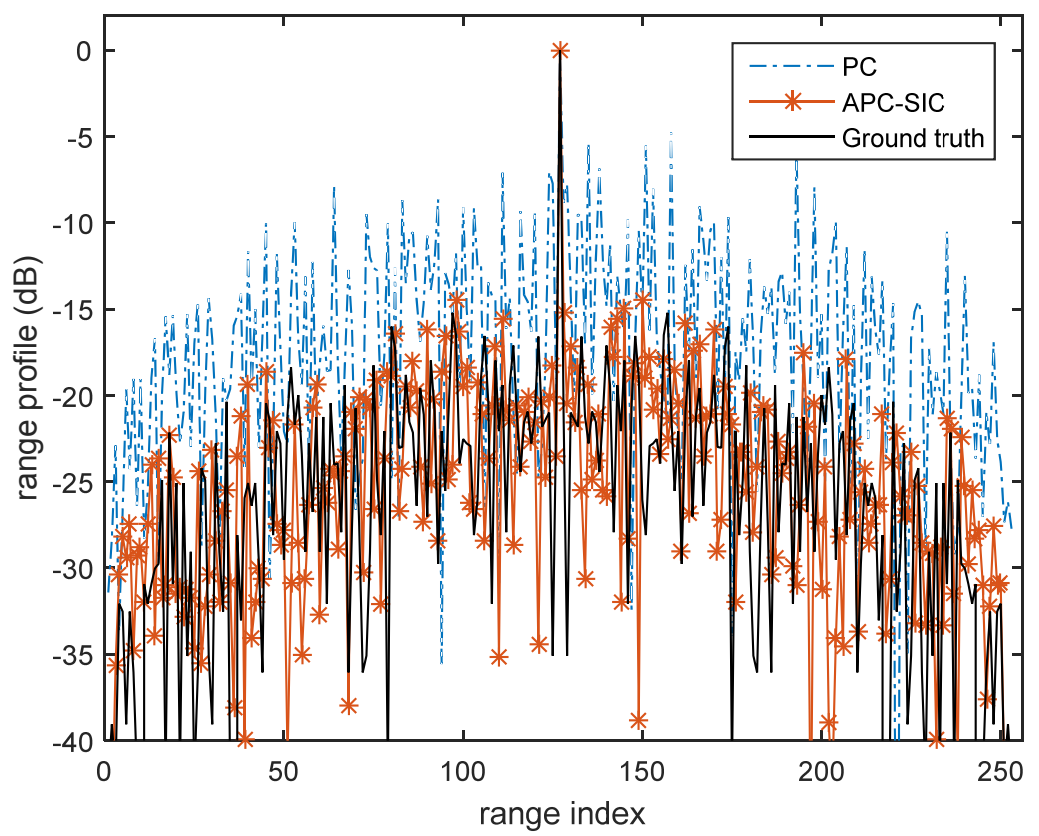

Fig.4. composite range profile of target $(\mathrm{M}=8)$.

It is shown in Fig.5 that for a scenario with $\mathrm{M}=16$ transmit antennas, the APC-SIC performs much better than the conventional pulse compression. The peak sidelobe of APC-SIC's output is $-10 \mathrm{~dB}$ when the peak sidelobe is $-2 \mathrm{~dB}$. 


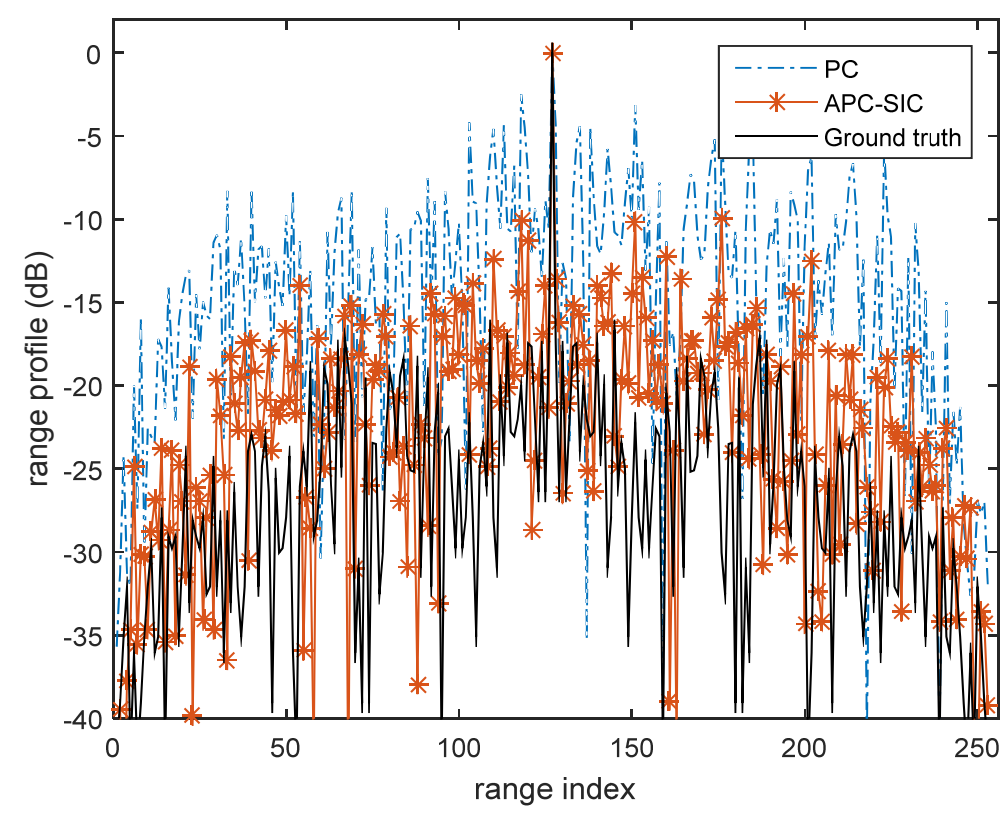

Fig.5. composite range profile of target $(\mathrm{M}=16)$.

\section{Conclusion}

The problem of suppress interference between transmitted signals of MIMO radar has been considered. Adaptive pulse compression based on SIC has been introduced to improve MIMO radar receivers. The essence of the proposed method is to subtract the estimation of other signals before pulse compression. Therefore the computation load is at the same level of conventional pulse compression. And it can effectively improve the output of pulse compression and suppress the sidelobe of range estimation, especially in the scenario that a large set of waveforms is applied by the MIMO radar.

\section{References}

[1] E. Fishler, A. Haimovich, R. Blum, D. Chizhik, L. Cimini, R. Valenzuela, MIMO radar: an idea whose time has come, in: Radar Conference, 2004. Proceedings of the IEEE, IEEE, 2004: pp. 7178.

[2] J. Li, P. Stoica, MIMO radar with colocated antennas, Signal Processing Magazine, IEEE. 24 (2007) 106-114.

[3] Chen, Chun-Yang, and Palghat P. Vaidyanathan, MIMO radar space-time adaptive processing using prolate spheroidal wave functions., Signal Processing, IEEE Transactions on. 56.2 (2008): 623-635.

[4] B. Liu, Z. He, J. Zeng, B. Liu, Polyphase orthogonal code design for MIMO radar systems, in: 2006 CIE International Conference on Radar, 2006: pp. 1-4.

[5] S.D. Blunt, K. Gerlach, Multistatic adaptive pulse compression, Aerospace and Electronic Systems, IEEE Transactions on. 42 (2006) 891-903.

[6] H.J. Blinchikoff, Range sidelobe reduction for the quadriphase codes, Aerospace and Electronic Systems, IEEE Transactions on. 32 (1996) 668-675.

[7] S.D. Blunt, K. Gerlach, Adaptive pulse compression via MMSE estimation, Aerospace and Electronic Systems, IEEE Transactions on. 42 (2006) 572-584. 